Algebras and Representation Theory manuscript No.

(will be inserted by the editor)

\title{
Regularity conditions for arbitrary Leavitt path algebras
}

\author{
Gene Abrams · Kulumani M. Rangaswamy
}

Received: date / Accepted: date

\begin{abstract}
We show that if $E$ is an arbitrary acyclic graph then the Leavitt path algebra $L_{K}(E)$ is locally $K$-matricial; that is, $L_{K}(E)$ is the direct union of subalgebras, each isomorphic to a finite direct sum of finite matrix rings over the field $K$. (Here an arbitrary graph means that neither cardinality conditions nor graph-theoretic conditions (e.g. row-finiteness) are imposed on $E$. These unrestrictive conditions are in contrast to the hypotheses used in much of the literature on this subject.) As a consequence we get our main result, in which we show that the following conditions are equivalent for an arbitrary graph $E$ : (1) $L_{K}(E)$ is von Neumann regular. $(2) L_{K}(E)$ is $\pi$-regular. (3) $E$ is acyclic. (4) $L_{K}(E)$ is locally $K$-matricial. (5) $L_{K}(E)$ is strongly $\pi$ regular. We conclude by showing how additional regularity conditions (unit regularity, strongly clean) can be appended to this list of equivalent conditions.
\end{abstract}

Keywords Leavitt path algebra; acyclic graph; von Neumann regular algebra

Mathematics Subject Classification (2000) Primary: 16S99, 16E50. Secondary: 16W50, 46L89

In this article we investigate Leavitt path algebras $L_{K}(E)$ over an arbitrary directed graph $E$ and an arbitrary field $K$. Here we require no restriction on either the size of the graph (i.e., vertex and edge sets may be of any cardinality), or on graph-theoretic constraints (i.e., no row-finiteness conditions are assumed). Our goal is to classify in ring-theoretic terms the Leavitt path algebras of the form $L_{K}(E)$ where $E$ is acyclic. A useful tool in our study is a construction presented in Proposition 1 which enables us to realize $L_{K}(E)$ as a directed union of subalgebras, each of which is a Leavitt path algebra of a suitable finite graph. An effective use of Proposition 1 leads to our main result, Theorem 1 in which we establish the equivalence of these conditions for an arbitrary directed graph $E$ and field $K:(1) L_{K}(E)$ is von Neumann regular. (2)

Gene Abrams and Kulumani M. Rangaswamy

Department of Mathematics

University of Colorado at Colorado Springs

Colorado Springs, Colorado, 80933 U.S.A.

E-mail: abrams@math.uccs.edu krangasw@uccs.edu

Corresponding author: G. Abrams 7192623182(v) 7192623605(f) 
$L_{K}(E)$ is $\pi$-regular. (3) $E$ is acyclic. (4) $L_{K}(E)$ is locally $K$-matricial (i.e., $L_{K}(E)$ is a direct union of subalgebras, each of which is isomorphic to a finite direct sum of finite matrix rings over $K)$. (5) $L_{K}(E)$ is strongly $\pi$-regular. We conclude the article by discussing various additional ring-theoretic conditions which in the context of Leavitt path algebras are equivalent to $E$ being acyclic.

We begin by giving a terse reminder of the germane definitions. For a more complete description and discussion, see e.g. 2] or 8. A (directed) graph $E=\left(E^{0}, E^{1}, r, s\right)$ consists of two sets $E^{0}, E^{1}$ and functions $r, s: E^{1} \rightarrow E^{0}$. (The sets $E^{0}$ and $E^{1}$ are allowed to be of arbitrary cardinality.) The elements of $E^{0}$ are called vertices and the elements of $E^{1}$ edges. A path $\mu$ in a graph $E$ is a sequence of edges $\mu=e_{1} \ldots e_{n}$ such that $r\left(e_{i}\right)=s\left(e_{i+1}\right)$ for $i=1, \ldots, n-1$. In this case, $s(\mu):=s\left(e_{1}\right)$ is the source of $\mu, r(\mu):=r\left(e_{n}\right)$ is the range of $\mu$, and $n$ is the length of $\mu$. We view the elements of $E^{0}$ as paths of length 0 . If $\mu=e_{1} \ldots e_{n}$ is a path in $E$, and if $v=s(\mu)=r(\mu)$ and $s\left(e_{i}\right) \neq s\left(e_{j}\right)$ for every $i \neq j$, then $\mu$ is called a cycle based at $v$. If $s^{-1}(v)$ is a finite set for every $v \in E^{0}$, then the graph $E$ is called row-finite.

Definition 1 Let $E$ be any directed graph, and $K$ any field. The Leavitt path $K$ algebra $L_{K}(E)$ of $E$ with coefficients in $K$ is the $K$-algebra generated by a set $\{v \mid v \in$ $\left.E^{0}\right\}$ of pairwise orthogonal idempotents, together with a set of variables $\left\{e, e^{*} \mid e \in\right.$ $\left.E^{1}\right\}$, which satisfy the following relations:

(1) $s(e) e=e r(e)=e$ for all $e \in E^{1}$.

(2) $r(e) e^{*}=e^{*} s(e)=e^{*}$ for all $e \in E^{1}$.

(3) (CK1) $e^{*} e^{\prime}=\delta_{e, e^{\prime}} r(e)$ for all $e, e^{\prime} \in E^{1}$.

(4) (CK2) $v=\sum_{\left\{e \in E^{1} \mid s(e)=v\right\}} e e^{*}$ for every vertex $v \in E^{0}$ having $1 \leq\left|s^{-1}(v)\right|<$ $\infty$

For any $F \subseteq E^{1}$ the set $\left\{e^{*} \mid e \in F\right\}$ will be denoted by $F^{*}$. We let $r\left(e^{*}\right)$ denote $s(e)$, and we let $s\left(e^{*}\right)$ denote $r(e)$. If $\mu=e_{1} \ldots e_{n}$ is a path, then we denote by $\mu^{*}$ the element $e_{n}^{*} \ldots e_{1}^{*}$ of $L_{K}(E)$.

Many well-known algebras arise as the Leavitt path algebra of a graph. For instance, the classical Leavitt algebras $L_{K}(1, n)$ for $n \geq 2$ arise as the algebras $L_{K}\left(R_{n}\right)$ where $R_{n}$ is the "rose with $n$ petals" graph described in Example1 below. (See e.g. 1, Section 3].) Also, for each $n \in \mathbb{N}=\{1,2, \ldots\}$, the full matrix ring $\mathrm{M}_{n}(K)$ arises as the Leavitt path algebra of the oriented $n$-line graph

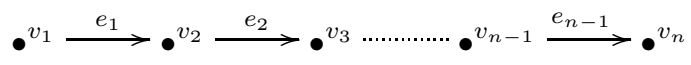

while the Laurent polynomial ring $K\left[x, x^{-1}\right]$ arises as the Leavitt path algebra of the "one vertex, one loop" graph

$$
\bullet v x
$$

A (possibly nonunital) ring $R$ is called a ring with local units in case for each finite subset $S \subseteq R$ there is an idempotent $e \in R$ with $S \subseteq e R e$. If $E$ is a graph for which $E^{0}$ is finite then we have $\sum_{v \in E^{0}} v$ is the multiplicative identity in $L_{K}(E)$; otherwise, $L_{K}(E)$ is a ring with a set of local units consisting of sums of distinct vertices. Conversely, if $L_{K}(E)$ is unital, then $E^{0}$ is finite. $L_{K}(E)$ is a $\mathbb{Z}$-graded $K$-algebra, spanned as a $K$ vector space by $\left\{p q^{*} \mid p, q\right.$ are paths in $\left.E\right\}$. (Recall that the elements of $E^{0}$ are viewed as paths of length 0 , so that this set includes elements of the form $v$ with $v \in E^{0}$.) In 
particular, for each $n \in \mathbb{Z}$, the degree $n$ component $L_{K}(E)_{n}$ is spanned by elements of the form $\left\{p q^{*} \mid \operatorname{length}(p)-\operatorname{length}(q)=n\right\}$. The degree of an element $x$, denoted $\operatorname{deg}(x)$, is the lowest number $n$ for which $x \in \bigoplus_{m \leq n} L_{K}(E)_{m}$. The $K$-linear extension of the assignment $p q^{*} \mapsto q p^{*}$ (for $p, q$ paths in $E$ ) yields an involution on $L_{K}(E)$, which we denote simply as *.

A subgraph $G$ of a graph $E$ is called complete in case, for each $v \in G^{0}$ having $1 \leq$ $\left|s_{G}^{-1}(v)\right|<\infty$, we have $s_{G}^{-1}(v)=s_{E}^{-1}(v)$. (In other words, a subgraph $G$ of $E$ is complete if, whenever $v \in G^{0}$ emits a nonzero, finite number of edges in $G$, then necessarily the subgraph $G$ contains all of the edges in $E$ emitted by $v$.) The natural inclusion map $L_{K}(G) \mapsto L_{K}(E)$ is a ring homomorphism precisely when $G$ is a complete subgraph of $E$, so that complete subgraphs of $E$ naturally give rise to subalgebras of $L_{K}(E)$. One of our main objectives in this article is to show how to construct subalgebras of $L_{K}(E)$ which need not arise in this way. This in turn will allow us to describe algebras of the form $L_{K}(E)$ as unions of subalgebras possessing various ring-theoretic properties, even in situations where $E$ lacks complete subgraphs possessing corresponding graphtheoretic properties. We achieve this objective in Proposition 11 The construction is based on an idea presented by Raeburn and Szymański in [13, Definition 1.1]; this work was brought to our attention by E. Pardo.

Definition 2 Let $E$ be a graph, and let $F$ be a finite set of edges in $E$. We define $s(F)$ (resp. $r(F)$ ) to be the sets of those vertices in $E$ which appear as the source (resp. range) vertex of at least one element of $F$. We define a graph $E_{F}$ as follows:

$$
\begin{gathered}
E_{F}^{0}=F \cup\left(r(F) \cap s(F) \cap s\left(E^{1} \backslash F\right)\right) \cup(r(F) \backslash s(F)), \\
E_{F}^{1}=\left\{(e, f) \in F \times E_{F}^{0} \mid r(e)=s(f)\right\} \cup[\{(e, r(e)) \mid e \in F \text { with } r(e) \in(r(F) \backslash s(F))\}], \\
\text { and where } s((x, y))=x, \quad r((x, y))=y \text { for any }(x, y) \in E_{F}^{1} .
\end{gathered}
$$

Note that, since $F$ is finite, the graph $E_{F}$ is finite (regardless of the size of $E$ ).

Remarks: 1. It is conventional to define $s(v)=v$ for each vertex $v$ in $E$. Because of that, the expression in rectangular brackets in Definition 2 for $E_{F}^{1}$ is redundant. However, we choose to keep this expression in the definition, as it makes the correspondence between $E_{F}^{1}$ and the set $G^{1}$ in the proof of Proposition 1 more transparent.

2. While the construction presented in Definition 2 is similar to that given in [13. Definition 1.1], there are indeed some significant differences. For instance, the construction of [13, Definition 1.1] requires that the graph $E$ has no sinks, while the construction presented here has no such stipulation. Additionally, even in situations where $E$ is a graph with no sinks and $F$ is a finite subset of $E^{1}$, the two constructions can in fact yield different corresponding graphs $E_{F}$. However, the underlying goal of each of the two constructions is the same, namely, to produce a subalgebra of a graph algebra which is isomorphic to the graph algebra of a finite graph.

Example 1 For clarity, we provide an example of the graph $E_{F}$ constructed in the previous definition. Let $E$ be the "rose with $n$-petals" graph

$$
E=
$$


Let $F=\left\{y_{1}\right\}$. Then $E_{F}^{0}=\left\{y_{1}\right\} \cup\{v\}$, and $E_{F}^{1}=\left\{\left(y_{1}, y_{1}\right),\left(y_{1}, v\right)\right\}$. Pictorially, $E_{F}$ is given by

$$
E_{F}=\left(y_{1}, y_{1}\right) \bigcup \bullet y_{1} \stackrel{\left(y_{1}, v\right)}{\longrightarrow} \bullet v
$$

This example indicates that various properties of the graph $E$ need not pass to the graph $E_{F}$. For instance, $E$ is cofinal, while $E_{F}$ is not. In particular, $L_{K}(E)$ is a simple algebra, while $L_{K}\left(E_{F}\right)$ is not. (See 2] for a more complete discussion.)

Our interest in the construction given in Definition 2 can be generally described as follows. We seek to place each finite set of elements taken from the Leavitt path algebra $L_{K}(E)$ inside a subalgebra of $L_{K}(E)$ which possesses certain 'finiteness' properties. In case $E$ is row-finite, by [5, Lemma 3.2] we can realize $L_{K}(E)$ as the direct union of subalgebras of the form $L_{K}\left(E_{i}\right)$ where each $E_{i}$ is a finite, complete subgraph of $E$. In the general case, however, we need not have such a description of $L_{K}(E)$. For instance, if $\aleph$ is an infinite cardinal, and $\operatorname{Clock}(\aleph)$ denotes the 'infinite clock' graph

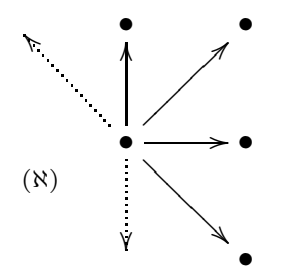

having $\aleph$ edges, then there are no nontrivial finite complete subgraphs of Clock $(\aleph)$.

Example 2 It will be instructive to consider the $E_{F}$ construction of Definition[2 within the infinite clock graph $E=\operatorname{Clock}(\aleph)$. So let $v$ denote the center vertex, let $f$ denote one of the edges, and let $w$ denote $r(f)$. Let $F=\{f\}$. Then $E_{F}^{0}=\{f\} \cup\{w\}$, while $E_{F}^{1}=\{(f, w)\}$. Thus $E_{F}$ is the graph

$$
E_{F}=\bullet \stackrel{(f, w)}{\longrightarrow} \bullet w
$$

with two vertices, and one edge connecting them. In particular, $L\left(E_{F}\right) \cong \mathrm{M}_{2}(K)$.

Although in general $E_{F}$ need not be a subgraph of $E$ (indeed, as seen in Example 1 $E_{F}$ may contain more vertices than does $E$ ), there is an important relationship between the Leavitt path algebras $L_{K}\left(E_{F}\right)$ and $L_{K}(E)$, as we now show.

Proposition 1 Let $F$ be a finite set of edges in a graph $E$. Then there is an algebra homomorphism $\theta: L_{K}\left(E_{F}\right) \rightarrow L_{K}(E)$ having the properties:

(1) $F \cup F^{*} \subseteq \operatorname{Im}(\theta)$.

(2) If $w \in r(F)$, then $w \in \operatorname{Im}(\theta)$.

(3) If $w \in E^{0}$ has $s_{E}^{-1}(w) \neq \emptyset$ and $s_{E}^{-1}(w) \subseteq F$, then $w \in \operatorname{Im}(\theta)$.

Proof We define subsets $G^{0}$ and $G^{1}$ of $L_{K}(E)$ as follows.

$$
\begin{aligned}
G^{0}= & \left\{e e^{*} \mid e \in F\right\} \\
& \cup\left\{v-\sum_{f \in F, s(f)=v} f f^{*} \mid v \in r(F) \cap s(F) \cap s\left(E^{1} \backslash F\right)\right\} \\
& \cup\{v \mid v \in r(F) \backslash s(F)\}
\end{aligned}
$$


and

$$
\begin{aligned}
G^{1}= & \left\{e f f^{*} \mid e, f \in F, s(f)=r(e)\right\} \\
& \cup\left\{e-\sum_{f \in F, s(f)=r(e)} e f f^{*} \mid r(e) \in r(F) \cap s(F) \cap s\left(E^{1} \backslash F\right)\right\} \\
& \cup\{e \in F \mid r(e) \in r(F) \backslash s(F)\} .
\end{aligned}
$$

We define $\theta: L_{K}\left(E_{F}\right) \rightarrow L_{K}(E)$ as follows.

There are three different types of vertices in $E_{F}$. If $w \in E_{F}^{0}$ has form $w=e \in F$, then define

$$
\theta(w)=e e^{*} .
$$

If $w \in E_{F}^{0}$ has form $w=v$ with $v \in r(F) \cap s(F) \cap s\left(E^{1} \backslash F\right)$, then define

$$
\theta(w)=v-\sum_{f \in F, s(f)=v} f f^{*} .
$$

If $w \in E_{F}^{0}$ has form $w=v$ with $v \in r(F) \backslash s(F)$, then define

$$
\theta(w)=w
$$

Note that in each case we have $\theta(w) \in G^{0}$.

There are three different types of edges in $E_{F}$. If $h \in E_{F}^{1}$ has form $h=(e, f)$ with $f \in F$, then define

$$
\theta(h)=e f f^{*} .
$$

If $h \in E_{F}^{1}$ has form $h=(e, r(e))$ with $r(e) \in r(F) \cap s(F) \cap s\left(E^{1} \backslash F\right)$, then define

$$
\theta(h)=e-\sum_{f \in F, s(f)=r(e)} e f f^{*} .
$$

If $h \in E_{F}^{1}$ has form $h=(e, r(e))$ with $r(e) \in r(F) \backslash s(F)$, then define

$$
\theta(h)=e .
$$

Note that in each case we have $\theta(h) \in G^{1}$.

For each $h \in E_{F}^{1}$ we define $\theta\left(h^{*}\right)=(\theta(h))^{*}$ in $L_{K}(E)$.

It is now a long, straightforward check to verify that $\theta$ is compatible with the four types of relations which define $L_{K}\left(E_{F}\right)$ (refer to Definition 1). As a representative example of the computations required here, we offer the following. Let $w \in E_{F}^{0}$ have the form $w=e \in F$. Then the (CK2) relation at $e$ in $L_{K}\left(E_{F}\right)$ is the equation

$$
\sum_{g \in E_{F}^{1}, s(g)=e} g g^{*}=e .
$$

But $s(g)=e$ in $E_{F}^{1}$ means $g=(e, f)$ where either $f \in F$ has $s(f)=r(e)$, or $g=(e, r(e))$ with $r(e) \in r(F) \cap s(F) \cap s\left(E^{1} \backslash F\right)$, or $g=(e, r(e))$ with $r(e) \in r(F) \backslash s(F)$. So the (CK2) relation at $e$ in $L_{K}\left(E_{F}\right)$ takes the form 


$$
\begin{aligned}
e= & \sum_{f \in F, s(f)=r(e)}(e, f)(e, f)^{*}+\sum_{w \in r(F) \cap s(F) \cap s\left(E^{1} \backslash F\right), w=r(e)}(e, w)(e, w)^{*} \\
& +\sum_{w \in r(F) \backslash s(F), w=r(e)}(e, w)(e, w)^{*} .
\end{aligned}
$$

Note that empty sums are interpreted as 0. Also, the final two summation expressions are in fact either singletons or empty, depending on whether $r(e) \in r(F) \cap s(F) \cap$ $s\left(E^{1} \backslash F\right)$ or $r(e) \in r(F) \backslash s(F)$.

We must show that the corresponding equation under $\theta$ holds in $L_{K}(E)$. In other words, we must show

$$
\begin{aligned}
e e^{*}= & \sum_{f \in F, s(f)=r(e)}\left(e f f^{*}\right)\left(e f f^{*}\right)^{*} \\
& +\sum_{w \in r(F) \cap s(F) \cap s\left(E^{1} \backslash F\right), w=r(e)}\left[e-\sum_{f \in F, s(f)=r(e)} e f f^{*}\right]\left[e-\sum_{f \in F, s(f)=r(e)} e f f^{*}\right]^{*} \\
& +\sum_{w \in r(F) \backslash s(F), w=r(e)} e e^{*} .
\end{aligned}
$$

There are two cases. If $r(e) \in r(F) \backslash s(F)$, then this equation simply becomes $e e^{*}=$ $e e^{*}$ and we are done. On the other hand, if $r(e) \in s(F)$, then note the second 'sum' $\sum_{w \in r(F) \cap s(F) \cap s\left(E^{1} \backslash F\right), w=r(e)}\left[e-\sum_{f \in F, s(f)=r(e)} e f f^{*}\right]\left[e-\sum_{f \in F, s(f)=r(e)} e f f^{*}\right]^{*}$ is in fact simply the single expression $\left[e-\sum_{f \in F, s(f)=r(e)} e f f^{*}\right]\left[e-\sum_{f \in F, s(f)=r(e)} \text { eff } f^{*}\right]^{*}$. So the right hand side is

$$
\begin{aligned}
& \sum_{f \in F, s(f)=r(e)}\left(e f f^{*}\right) f f^{*} e^{*}+\left[e-\sum_{f \in F, s(f)=r(e)} e f f^{*}\right]\left[e-\sum_{f \in F, s(f)=r(e)} e f f^{*}\right]^{*} \\
= & \sum_{f \in F, s(f)=r(e)} e f f^{*} e^{*}+\left[e e^{*}-\sum_{f \in F, s(f)=r(e)} e f f^{*} e^{*}\right] \quad(\text { by }(\mathrm{CK} 1) \text { and (CK2)) } \\
= & e e^{*} .
\end{aligned}
$$

In a similar manner one can verify the compatibility of $\theta$ with all the remaining relations which define $L_{K}\left(E_{F}\right)$. Thus we conclude that $\theta$ extends to an algebra homomorphism

$$
\theta: L_{K}\left(E_{F}\right) \rightarrow L_{K}(E)
$$

By definition we have $\operatorname{Im}(\theta)$ is the subalgebra of $L_{K}(E)$ generated by $G^{0}, G^{1},\left(G^{1}\right)^{*}$. It will be helpful later to note that for each $x \in G^{1} \cup\left(G^{1}\right)^{*}$ there exist $y, y^{\prime} \in G^{0}$ for which $y x y^{\prime}=x$. In particular, if an element $z \in L_{K}(E)$ is orthogonal to every element of $G^{0}$, then necessarily $z$ is orthogonal to every element in $\operatorname{Im}(\theta)$.

We are now in position to verify the three claimed properties of $\operatorname{Im}(\theta)$. For (1), we show that every $f \in F$ is contained in $\operatorname{Im}(\theta)$. Suppose first that $f \in F$ has $s_{E}^{-1}(r(f)) \subseteq$ 
$F$. If $s_{E}^{-1}(r(f))=\emptyset$, then $r(f) \in r(F) \backslash s(F)$ vacuously, so by definition $f \in G^{1} \subseteq \operatorname{Im}(\theta)$. On the other hand, if $s_{E}^{-1}(r(f)) \neq \emptyset$, then we have $f g g^{*} \in G^{1}$ for all $g \in F$ having $r(f)=s(g)$. But then by hypothesis this is the same as the collection of $g \in E^{1}$ having $r(f)=s(g)$. Thus we have $\left\{f g g^{*} \mid g \in E^{1}, s(g)=r(f)\right\} \subseteq \operatorname{Im}(\theta)$, so that in particular $\operatorname{Im}(\theta)$ contains $\sum_{g \in E^{1}, s(g)=r(f)} f g g^{*}=f \cdot \sum_{g \in E^{1}, s(g)=r(f)} g g^{*}=f \cdot r(f)=f$.

On the other hand, suppose that $f \in F$ has the property that $s_{E}^{-1}(r(f)) \nsubseteq F$. Then there are two possibilities. In the first case, $s_{E}^{-1}(r(f)) \neq \emptyset$ and $s_{E}^{-1}(r(f)) \cap F=\emptyset$. (In other words, there are edges in $E$ which are emitted from $r(f)$, but none of these edges are in $F$.) But then $r(f) \notin s(F)$, so that $f \in G^{1}$ by definition, so that $f \in \operatorname{Im}(\theta)$. In the second case, suppose $s_{E}^{-1}(r(f)) \cap F \neq \emptyset$. Then either we have $s_{E}^{-1}(r(f)) \subseteq F$ (in which case we are done by the previous paragraph), or we have $r(f) \in r(F) \cap s(F) \cap s\left(E^{1} \backslash F\right)$. In this situation we have $f g g^{*} \in G^{1} \subseteq \operatorname{Im}(\theta)$ for all $g \in F$ having $s(g)=r(f)$, so we in particular have $\sum_{g \in F, s(g)=r(f)} f g g^{*}$ in $\operatorname{Im}(\theta)$ as well. But by definition we also have the element $f-\sum_{g \in F, s(g)=r(f)} f g g^{*}$ in $G^{1} \subseteq \operatorname{Im}(\theta)$. Then

$$
f=\left(\sum_{g \in F, s(g)=r(f)} f g g^{*}\right)+\left(f-\sum_{g \in F, s(g)=r(f)} f g g^{*}\right) \in \operatorname{Im}(\theta) .
$$

Thus we conclude that $F \subseteq \operatorname{Im}(\theta)$. But for each $x \in \operatorname{Im}(\theta)$ we have $x^{*} \in \operatorname{Im}(\theta)$ by definition. Thus $F \cup F^{*} \subseteq \operatorname{Im}(\theta)$, thereby establishing (1).

In particular, if $w=r(f)$ for $f \in F$, then $w=f^{*} f \in \operatorname{Im}(\theta)$, yielding (2). For (3), suppose $s^{-1}(w) \neq \emptyset$, and $s^{-1}(w) \subseteq F$. Then each $f f^{*}$ for $f \in E^{1}$ having $s(f)=w$ is in $G^{0}$, so that $\sum_{f \in E^{1}, s(f)=w} f f^{*}$ is in $\operatorname{Im}(\theta)$. But this last sum is precisely $w$ by (CK2).

We remark here that for $\theta$ as given in Proposition [1, $\theta(w) \neq 0$ for all three possible types of $w \in E_{F}^{0}$. (That $\theta(w) \neq 0$ in case $w \in r(F) \cap s(F) \cap s\left(E^{1} \backslash F\right)$ hinges on the fact that there exists $g \in E^{1} \backslash F$ having $s(g)=w$.) This in turn will allow us to conclude, in certain situations (including the situation where $E$ is acyclic), that $\theta$ is in fact a monomorphism. (See e.g. [1].) However, we will not utilize this additional property of $\theta$ in the sequel.

With Proposition 1 in hand, we now construct the subalgebras of $L_{K}(E)$ which play the central role in our main result, Theorem 1 .

\section{The Subalgebra Construction}

Let $E$ be any graph, $K$ any field, and $\left\{a_{1}, a_{2}, \ldots, a_{\ell}\right\}$ any finite subset of nonzero elements of $L_{K}(E)$. For each $1 \leq r \leq \ell$ write

$$
a_{r}=k_{c_{1}} v_{c_{1}}+k_{c_{2}} v_{c_{2}}+\ldots+k_{c_{j(r)}} v_{c_{j(r)}}+\sum_{i=1}^{t(r)} k_{r_{i}} p_{r_{i}} q_{r_{i}}^{*}
$$

where each $k_{j}$ is a nonzero element of $K$, and, for each $1 \leq i \leq t(r)$, at least one of $p_{r_{i}}$ or $q_{r_{i}}$ has length at least 1 . (That such a representation for each $a_{r}$ exists follows from properties of $L_{K}(E)$ mentioned previously.) Let $F$ denote the (necessarily finite) set of 
those edges in $E$ which appear in the representation of some $p_{r_{i}}$ or $q_{r_{i}}, 1 \leq r_{i} \leq t(r)$, $1 \leq r \leq \ell$. Now consider the set

$$
S=\left\{v_{c_{1}}, v_{c_{2}}, \ldots, v_{c_{j(r)}} \mid 1 \leq r \leq \ell\right\}
$$

of vertices which appear in the displayed description of $a_{r}$ for some $1 \leq r \leq \ell$. We partition $S$ into subsets as follows:

$$
S_{1}=S \cap r(F),
$$

and, for the remaining vertices $T=S \backslash S_{1}$, we define

$$
\begin{gathered}
S_{2}=\left\{v \in T \mid s_{E}^{-1}(v) \subseteq F \text { and } s_{E}^{-1}(v) \neq \emptyset\right\} \\
S_{3}=\left\{v \in T \mid s_{E}^{-1}(v) \cap F=\emptyset\right\} \\
S_{4}=\left\{v \in T \mid s_{E}^{-1}(v) \cap F \neq \emptyset \text { and } s_{E}^{-1}(v) \cap\left(E^{1} \backslash F\right) \neq \emptyset\right\} .
\end{gathered}
$$

Let $E_{F}$ be the graph as constructed in Definition 2 corresponding to this set $F$, and let $\theta: L_{K}\left(E_{F}\right) \rightarrow L_{K}(E)$ be the homomorphism described in Proposition 1

Definition 3 Let $E$ be any graph, $K$ any field, and $\left\{a_{1}, a_{2}, \ldots, a_{\ell}\right\}$ any finite subset of nonzero elements of $L_{K}(E)$. Consider the notation presented in The Subalgebra Construction. We define $B\left(a_{1}, a_{2}, \ldots, a_{\ell}\right)$ to be the $K$-subalgebra of $L_{K}(E)$ generated by the set $\operatorname{Im}(\theta) \cup S_{3} \cup S_{4}$. That is,

$$
B\left(a_{1}, a_{2}, \ldots, a_{\ell}\right)=<\operatorname{Im}(\theta), S_{3}, S_{4}>.
$$

Proposition 2 Let $E$ be any graph, $K$ any field, and $\left\{a_{1}, a_{2}, \ldots, a_{\ell}\right\}$ any finite subset of nonzero elements of $L_{K}(E)$. Let $F$ denote the subset of $E^{1}$ presented in The Subalgebra Construction. For $w \in S_{4}$ let $u_{w}$ denote the element $w-\sum_{f \in F, s(f)=w} f f^{*}$ of $L_{K}(E)$. Then

(1) $\left\{a_{1}, a_{2}, \ldots, a_{\ell}\right\} \subseteq B\left(a_{1}, a_{2}, \ldots, a_{\ell}\right)$.

(2) $B\left(a_{1}, a_{2}, \ldots, a_{\ell}\right)=\operatorname{Im}(\theta) \oplus\left(\oplus_{v_{i} \in S_{3}} K v_{i}\right) \oplus\left(\oplus_{w_{j} \in S_{4}} K u_{w_{j}}\right)$.

(3) The collection $\left\{B(S) \mid S \subseteq L_{K}(E)\right.$, S finite $\}$ is an upward directed set of subalgebras of $L_{K}(E)$.

(4) $L_{K}(E)=\lim _{\left\{S \subseteq L_{K}(E), S \text { finite }\right\}} B(S)$.

Proof (1) By Proposition 11 we have that $F \cup F^{*} \cup S_{1} \cup S_{2} \subseteq B\left(a_{1}, a_{2}, \ldots, a_{\ell}\right)$. Since $S_{3} \cup S_{4} \subseteq B\left(a_{1}, a_{2}, \ldots, a_{\ell}\right)$ by construction, (1) follows.

(2) Since the $\left\{v_{i}\right\}$ and $\left\{u_{w_{j}}\right\}$ are pairwise orthogonal idempotents we immediately get that $\sum_{v_{i} \in S_{3}} K v_{i}=\oplus_{v_{i} \in S_{3}} K v_{i}$, and that $\sum_{w_{j} \in S_{4}} K u_{w_{j}}=\oplus_{w_{j} \in S_{4}} K u_{w_{j}}$. We now establish that the three indicated summands are mutually orthogonal, which will establish that the sum $\operatorname{Im}(\theta)+\left(\oplus_{v_{i} \in S_{3}} K v_{i}\right)+\left(\oplus_{w_{j} \in S_{4}} K u_{w_{j}}\right)$ is direct. Let $v \in S_{3}$. Then by definition $v$ is neither the source vertex nor range vertex for any of the elements in $F$. In particular, the one dimensional subalgebra $K v$ of $L_{K}(E)$ clearly annihilates all the elements of $G^{0}$; as noted previously, this suffices to yield that $K v$ indeed annihilates $\operatorname{Im}(\theta)$. But for any $w \in S_{4}$ we have that $u_{w}=w-\sum_{f \in F, s(f)=w} f f^{*}$ is orthogonal in $L_{K}(E)$ to $v$, since $S_{3} \cap S_{4}=\emptyset$.

So we have shown that $\oplus_{v_{i} \in S_{3}} K v_{i} \cap\left[\operatorname{Im}(\theta)+\oplus_{w_{j} \in S_{4}} K u_{w_{j}}\right]=\{0\}$. Thus we need only show that $\operatorname{Im}(\theta) \cap K u_{w}=\{0\}$ for all $w \in S_{4}$, which we establish by showing 
$K u_{w} \cdot \operatorname{Im}(\theta)=\operatorname{Im}(\theta) \cdot K u_{w}=\{0\}$ and using the fact that each $u_{w}$ is idempotent. Choose any such $w$. Since by definition $w \notin r(F)$ we have that $u_{w}=w-\sum_{f \in F, s(f)=w} f f^{*}$ is orthogonal in $L_{K}(E)$ to elements of $G^{0}$ of the form $w^{\prime}-\sum_{g \in F, s(g)=w^{\prime}} g g^{*}$ for $w^{\prime} \in r(F) \cap s(F) \cap s\left(E^{1} \backslash F\right)$. Similarly, $u_{w}$ is orthogonal in $L_{K}(E)$ to $r(e)$ for each $e \in F$. Now suppose $e e^{*} \in G^{0}$ with $e \in F$. If $s(e) \neq w$ then $w-\sum_{f \in F, s(f)=w} f f^{*}$ is clearly orthogonal to $e e^{*}$. On the other hand, if $s(e)=w$ then $u_{w} \cdot e e^{*}=(w-$ $\left.\sum_{f \in F, s(f)=w} f f^{*}\right) \cdot e e^{*}=w e e^{*}-\sum_{f \in F, s(f)=w} f f^{*} e e^{*}=e e^{*}-e e^{*}=0$, with the final simplification occurring because $f f^{*} e e^{*}=e e^{*}$ for $e=f$, and $f f^{*} e e^{*}=0$ otherwise by (CK1). Similarly, we have $e e^{*} \cdot u_{w}=0$. We conclude that the indicated sums are direct.

We now show that the direct sum in fact equals $B\left(a_{1}, a_{2}, \ldots, a_{\ell}\right)$. By construction, it suffices to show that $u_{w} \in B\left(a_{1}, a_{2}, \ldots, a_{\ell}\right)$ for all $w \in S_{4}$, and that $w \in \operatorname{Im}(\theta) \oplus$ $\left(\oplus_{v_{i} \in S_{3}} K v_{i}\right) \oplus\left(\oplus_{w_{j} \in S_{4}} K u_{w_{j}}\right)$ for all $w \in S_{4}$. But each of these inclusions follow directly by noting that, for each $w \in S_{4}$ and each $f \in F$ having $s(f)=w$, we have $f f^{*} \in \operatorname{Im}(\theta)$ by definition.

(3) As noted previously, $E_{F}$ is a finite graph for each finite subset $F$ of $E^{1}$. In particular, $L_{K}\left(E_{F}\right)$ is a finitely generated $K$-algebra (with generating set $E_{F}^{0} \cup E_{F}^{1} \cup$ $\left.\left(E_{F}^{1}\right)^{*}\right)$. This in turn implies that $\operatorname{Im}(\theta)$, and hence $B(S)$, is a finitely generated $K$ algebra for each finite set $S$ of $L_{K}(E)$. In particular, if $S_{1}$ and $S_{2}$ are finite subsets of $L_{K}(E)$, we let $T_{1}$ (resp. $\left.T_{2}\right)$ denote a finite set of generators of $B\left(S_{1}\right)\left(\operatorname{resp} . B\left(S_{2}\right)\right)$. If $T=T_{1} \cup T_{2}$, then it is clear by construction that $B\left(S_{1}\right) \cup B\left(S_{2}\right) \subseteq B(T)$.

(4) now follows immediately from (1) and (3).

As noted previously, various properties of the graph $E$ need not pass to the graph $E_{F}$. However,

Lemma 1 Let $E$ be any acyclic graph, and $F$ any finite subset of $E^{1}$. Then $E_{F}$ is acyclic.

Proof By contradiction, we show that the existence of a closed path in $E_{F}$ necessarily yields a closed path in $E$. By definition, a closed path in $E_{F}$ is of the form $\left(e_{1}, e_{2}\right),\left(e_{2}, e_{3}\right), \ldots,\left(e_{n}, e_{1}\right)$ where $\left(e_{i}, e_{i+1}\right) \in E_{F}^{1}$. Now it is straightforward to show that the indicated sequence of edges in $E_{F}$ yields a sequence $e_{1}, e_{2}, \ldots, e_{n}$ in $E^{1}$ having the desired property.

We recall now some ideas which play central roles in our main result. For additional information about these concepts, see for example [9], 10, and [1].

Definition 4 Let $R$ be a (not necessarily unital) ring.

(1) $R$ is called von Neumann regular in case for every $x \in R$ there exists $y \in R$ such that $x=x y x$.

(2) $R$ is called $\pi$-regular in case for every $x \in R$ there exists $y \in R$ and $n \in \mathbb{N}$ for which $x^{n}=x^{n} y x^{n}$.

(3) $R$ is called left (resp. right) $\pi$ - regular if for each $a \in R$ there exists $n \in \mathbb{N}$ and $b \in R$ such that $a^{n}=b a^{n+1}$ (resp. $a^{n}=a^{n+1} b$ ). (For rings with local units, this is equivalent to saying that the descending chain of left ideals $R a \supseteq R a^{2} \supseteq \ldots \supseteq R a^{k} \supseteq \ldots$ (resp. right ideals $a R \supseteq a^{2} R \supseteq \ldots \supseteq a^{k} R \supseteq \ldots$ ) becomes stationary after finitely many terms.)

(4) $R$ is called strongly $\pi$-regular if its both left and right $\pi$-regular. 
Clearly any von Neumann regular ring is $\pi$-regular. Conversely, the ring $\mathbb{Z} / 4 \mathbb{Z}$ provides an easy example of a ring which is $\pi$-regular but not von Neumann regular (since $\overline{2}$ has no von Neumann regular inverse).

By [7, Lemma 6], if $R$ is a unital strongly $\pi$-regular ring then for every element $a \in R$ there is a positive integer $n$ and an element $x \in R$ such that $a x=x a$ and $a^{n+1} x=a^{n}=x a^{n+1}$. (We will show below that this result holds for rings with local units as well.) From this property it is then easy to show that if $R$ is strongly $\pi$-regular, then $R$ is $\pi$-regular. Conversely, the ring $R=\operatorname{End}_{K}(V)$ of all linear transformations of an infinite dimensional vector space $V$ over a field $K$ provides an example of a ring which is $\pi$-regular (in fact, von Neumann regular), but not strongly $\pi$-regular. (Indeed, if $\alpha: V \rightarrow V$ is the shift transformation given by $\alpha\left(K_{1}\right)=0$ and $\alpha\left(K_{i+1}\right)=K_{i}$ for all $i>1$, then for any $n$, $\operatorname{ker} \alpha^{n}=\oplus_{i=1}^{n} K_{i}$ and so $\alpha^{n} \neq \beta \alpha^{n+1}$ for any $n$.)

Lemma 2 Let $R$ be a ring with local units. Then $R$ is strongly $\pi$-regular ring if and only if for every nonzero idempotent $v$ of $R$, the subring $v R v$ is strongly $\pi$-regular.

Proof Assume $\mathrm{R}$ is strongly $\pi$-regular. Pick $a \in v R v$. By hypothesis there exists $b \in R$ with $a^{n}=a^{n+1} b$, and there exists $c \in R$ with $a^{m}=c a^{m+1}$. But $v a v=v$, so $v a^{n} v=a^{n}$ and $v a^{n+1} v=a^{n+1}$. Thus, multiplying both sides of the equation $a^{n}=a^{n+1} b$ by $v$, we get $a^{n}=v a^{n+1} v b v=a^{n+1} v b v$. Since $v b v \in v R v$ we have shown that $a^{n}=a^{n+1} b^{\prime}$ for some $b^{\prime} \in v R v$. A similar computation yields that $a^{m}=c^{\prime} a^{m+1}$ inside $v R v$.

Conversely, pick $a \in R$. Then $a \in v R v$ for some idempotent $v$ by definition of set of local units. So there exist $b$ and $c$ in $v R v$, and hence in $R$, with the appropriate properties.

Although the properties von Neumann regular, strongly $\pi$-regular, and $\pi$-regular are in general not equivalent, as one consequence of Theorem 1 we conclude that these properties are indeed equivalent in the context of Leavitt path algebras.

We are now in position to establish our main result.

Theorem 1 Let $E$ be an arbitrary graph, and let $K$ be any field. The following are equivalent.

(1) $L_{K}(E)$ is von Neumann regular.

(2) $L_{K}(E)$ is $\pi$-regular.

(3) $E$ is acyclic.

(4) $L_{K}(E)$ is locally $K$-matricial; that is, $L_{K}(E)$ is the direct union of subrings, each of which is isomorphic to a finite direct sum of finite matrix rings over $K$.

(5) $L_{K}(E)$ is strongly $\pi$-regular.

Proof $(1) \Rightarrow(2)$ is immediate.

$(2) \Rightarrow(3)$. By contradiction, suppose $c$ is a cycle in $E$, and let $v=s(c)=r(c)$. We show that $v+c$ has no $\pi$-regular inverse in $L_{K}(E)$.

Let $\gamma$ denote $v+c$. Suppose there exist $\beta \in L_{K}(E), n \in \mathbb{N}$ such that $\gamma^{n} \beta \gamma^{n}=\gamma^{n}$. Note that, since $\gamma v=\gamma=v \gamma$, we have $\gamma^{n} v \beta v \gamma^{n}=\gamma^{n}$. Then $\alpha=v \beta v$ satisfies $\gamma^{n} \alpha \gamma^{n}=\gamma^{n}$ and $v \alpha v=\alpha$. Moreover, $\alpha v=v \alpha=\alpha$.

Write $\alpha$ as a graded sum $\alpha=\sum_{i=M}^{N} a_{i}$ where $a_{M} \neq 0, a_{N} \neq 0, \operatorname{deg}\left(a_{i}\right)=i$ for all nonzero $a_{i}$ having $M \leq i \leq N$, and $a_{i}=0$ if $i>N$ or $i<M$. Since $\operatorname{deg}(v)=0$, the 
equation $\alpha v=v \alpha$ implies that $a_{i} v=v a_{i}=a_{i}$ for all $i$. Now expanding the equation $\gamma^{n} \alpha \gamma^{n}=\gamma^{n}$, we obtain

$$
\left(v+\sum_{k=1}^{n}\left(\begin{array}{l}
n \\
k
\end{array}\right) c^{k}\right)\left(\sum_{i=M}^{N} a_{i}\right)\left(v+\sum_{k=1}^{n}\left(\begin{array}{l}
n \\
k
\end{array}\right) c^{k}\right)=v+\sum_{k=1}^{n}\left(\begin{array}{l}
n \\
k
\end{array}\right) c^{k} .
$$

Equating the lowest degree terms on both sides, we get $v a_{M} v=v$, so that $a_{M}=v$. Since $\operatorname{deg}(v)=0$, we conclude that $M=0$, and that $a_{0}=v$. Thus $\alpha=\sum_{i=0}^{N} a_{i}$. Let $\operatorname{deg}(c)=s>0$. Now every term other than the first on the right hand side has degree $k s$ for some positive integer $k \leq n$, and so equating the corresponding graded components on both sides, we conclude that $a_{i}=0$ if $i$ is not a multiple of $s$.

We establish by induction that $a_{k s}=f_{k}(c)$ for each $k \in \mathbb{N}$, where $f_{k}(c)$ is a polynomial in $c$ with integer coefficients. For $k=1$, by equating the degree $s$ components on both sides we obtain

$$
v a_{s} v+\left(\begin{array}{l}
n \\
1
\end{array}\right) c a_{0}+a_{0}\left(\begin{array}{l}
n \\
1
\end{array}\right) c=\left(\begin{array}{l}
n \\
1
\end{array}\right) c .
$$

This implies that $a_{s}=-\left(\begin{array}{l}n \\ 1\end{array}\right) c$, an integral polynomial in $c$. Now suppose $t>1$, and suppose $a_{k s}=f_{k}(c)$, an integral polynomial in $c$ for all $1 \leq k<t$. We expand the previously displayed equation, and equate the degree $t s$ terms of both sides. This yields

$$
\begin{aligned}
a_{t s} & +\left(\begin{array}{l}
n \\
1
\end{array}\right) c\left[a_{(t-1) s}+a_{(t-2) s}\left(\begin{array}{l}
n \\
1
\end{array}\right) c+a_{(t-3) s}\left(\begin{array}{l}
n \\
2
\end{array}\right) c^{2}+\ldots+a_{0}\left(\begin{array}{c}
n \\
t-1
\end{array}\right) c^{t-1}\right] \\
& +\left(\begin{array}{l}
n \\
2
\end{array}\right) c^{2}\left[a_{(t-2) s}+a_{(t-3) s}\left(\begin{array}{l}
n \\
1
\end{array}\right) c+\ldots+a_{0}\left(\begin{array}{c}
n \\
t-2
\end{array}\right) c^{t-2}\right] \\
& +\left(\begin{array}{l}
n \\
3
\end{array}\right) c^{3}\left[a_{(t-3) s}+a_{(t-4) s}\left(\begin{array}{l}
n \\
1
\end{array}\right) c+\ldots+a_{0}\left(\begin{array}{c}
n \\
t-3
\end{array}\right) c^{t-3}\right] \\
& +\ldots+\left(\begin{array}{c}
n \\
t
\end{array}\right) c^{t} a_{0} \\
= & \left(\begin{array}{c}
n \\
t
\end{array}\right) c^{t}
\end{aligned}
$$

Substituting for $a_{s}, \ldots, a_{(t-1) s}$ as allowed by the induction hypothesis and solving for $a_{t s}$, we obtain $a_{t s}=f_{t}(c)$, a polynomial in $c$ with integer coefficients.

In particular, we conclude that every homogeneous component $a_{i}$ of $\alpha$ commutes with $c$ in $L_{K}(E)$. This yields that $c \alpha=\alpha c$. But then the equation $(v+c)^{n} \alpha(v+c)^{n}=$ $(v+c)^{n}$ becomes

$$
\alpha(v+c)^{2 n}=(v+c)^{n}
$$

But this is impossible, as follows. Since each $a_{i}$ is a polynomial in $c$ with integer coefficients, we have $a_{i} c^{r} \neq 0$ for all $r \in \mathbb{N}$. Let $i$ be maximal with the property that $a_{i}(v+c)^{2 n} \neq 0$. (Such $i$ exists, since $a_{0}=v$ has this property.) Then the left hand side contains terms of degree $2 s n+i$ (namely, $a_{i} c^{2 n}$ ), while the maximum degree of terms on the right hand side is $n s$. 
$(3) \Rightarrow(4)$. We assume $E$ is acyclic. Let $\left\{B(S) \mid S \subseteq L_{K}(E), S\right.$ finite $\}$ be the collection of subalgebras of $L_{K}(E)$ indicated in Proposition 2(3). By Proposition 2(4), it suffices to show that each such $B(S)$ is of the indicated form. But by Proposition 2(2), $B(S)=\operatorname{Im}(\theta) \oplus\left(\oplus_{v_{i} \in S_{3}} K v_{i}\right) \oplus\left(\oplus_{w_{j} \in S_{4}} K u_{w_{j}}\right)$. Since terms appearing in the second and third summands are clearly isomorphic as algebras to $K \cong \mathrm{M}_{1}(K)$, it suffices to show that $\operatorname{Im}(\theta)$ is isomorphic to a finite direct sum of finite matrix rings over $K$. Since $E$ is acyclic, by Lemma 1 we have that $E_{F}$ is acyclic. But $E_{F}$ is always finite by definition, so we have by [3, Proposition 3.5] that $L\left(E_{F}\right) \cong \oplus_{i=1}^{\ell} \mathrm{M}_{m_{i}}(K)$ for some $m_{1}, \ldots, m_{\ell}$ in $\mathbb{N}$. Since each $\mathrm{M}_{m_{i}}(K)$ is a simple ring, we have that any homomorphic image of $L_{K}\left(E_{F}\right)$ must have this same form. So we get that $\operatorname{Im}(\theta) \cong \oplus_{i=1}^{L} \mathrm{M}_{m_{i}}(K)$ for some $m_{1}, \ldots, m_{L}$ in $\mathbb{N}$, and we are done. (As remarked previously, since $\theta$ is in fact an isomorphism we have $L=\ell$.)

$(4) \Rightarrow(1)$. It is well known that any algebra of the form $\oplus_{i=1}^{\ell} \mathrm{M}_{m_{i}}(K)$ is von Neumann regular. But every element of $L_{K}(E)$ is contained in a subalgebra of $L_{K}(E)$ of this form, so that every element of $L_{K}(E)$ thereby has a von Neumann regular inverse.

$(4) \Rightarrow(5)$. Suppose $L_{K}(E)$ is locally $K$-matricial. So every element $a \in L_{K}(E)$ is contained in a subring $S \cong \oplus_{i=1}^{\ell} \mathrm{M}_{m_{i}}(K)$. As any such $S$ is a unital left (resp. right) artinian ring, there is a $b \in S$ and a positive integer $n$ such $a^{n}=b a^{n+1}$ (resp. $\left.a^{n}=a^{n+1} b\right)$.

$(5) \Rightarrow(2)$ By Lemma 2 we have that each $a \in L_{K}(E)$ is contained in a strongly $\pi$-regular unital subring of the form $v L_{K}(E) v$ for some $v=v^{2} \in L_{K}(E)$. Then by 7 , Lemma 6] there is a positive integer $n$ and an element $x \in v L_{K}(E) v$ such that $a x=x a$ and $a^{n+1} x=a^{n}=x a^{n+1}$. Now iterating the substitution $a^{n}=a^{n+1} x=a a^{n} x=$ $a\left(a^{n+1} x\right) x=a^{n+2} x^{2}$ we get $a^{n}=a^{2 n} x^{n}$, which using $a x=x a$ gives $a^{n}=a^{n} x^{n} a^{n}$, which yields $(2)$.

We record the following consequence of Theorem 1 in part because it demonstrates the independence of our results from any cardinality restrictions or graph-theoretic restrictions (e.g. row-finiteness) on the graphs.

Example 3 Let $\aleph$ be any cardinal, and let $\operatorname{Clock}(\aleph)$ be the infinite clock graph having $\aleph$ edges. Then for any field $K$, the Leavitt path algebra $L_{K}(\operatorname{Clock}(\aleph))$ is von Neumann regular. In addition, $L_{K}(\operatorname{Clock}(\aleph))$ is locally $K$-matricial.

It is worth noting that the locally $K$-matricial nature of $L_{K}(\operatorname{Clock}(\aleph))$ does not stem from a consideration of the finite complete subgraphs of Clock( $\aleph)$, since as noted previously Clock $(\aleph)$ contains no such nontrivial subgraphs.

As a second consequence of Theorem 1, we see that the $\operatorname{ring} R=\operatorname{End}_{K}(V)$ of all linear transformations of an infinite dimensional vector space $V$ over a field $K$ cannot be represented as $L_{K}(E)$ for any graph $E$, since $R$ is von Neumann regular but not strongly $\pi$-regular (as noted earlier). Similarly, let $V$ be a vector space of uncountable dimension over a field $K$ and let $S$ be the (nonunital) subring of $\operatorname{End}_{K}(V)$ consisting of those linear transformations whose images are of at most countable dimension. Then $S$ is a von Neumann regular ring with local units. However, $S$ is not strongly $\pi$-regular, so again invoking Theorem 1 we have that $S$ cannot be represented as the Leavitt path algebra of any graph $E$. 
We conclude this article by analyzing two additional "regularity" properties of a ring. We recall the definitions of some ring-theoretic terms.

Definition 5 Let $R$ be a unital ring.

(1) $R$ is called clean if each $a \in R$ is of the form $a=e+u$ where $e$ is an idempotent and $u$ is a (two-sided) unit. If in addition $a R \cap e R=0$, we say $R$ is a special clean ring. A clean ring $R$ is said to be strongly clean if in the above definition we can choose $e$ and $u$ which commute.

(2) $R$ is called unit regular in case for each $a \in R$ there exists a (two-sided) unit $u \in R$ such that $a u a=a$. In particular, every unit regular ring is von Neumann regular.

Additional information about clean rings can be found in 11, while additional information about unit regular rings can be found in 9. The properties "clean" and "unit regular" are exemplified by matrix rings. Indeed if $R$ is the ring of $n \times n$ matrices over a field, then $R$ is both unit regular [9, page 38] and strongly clean [11, Theorem 4.1]. By [6, Theorem 1], a unital ring $R$ is unit regular if and only if $R$ is a special clean ring; in particular, any ring of the form $\mathrm{M}_{n}(K)$ for $K$ a field and $n \in \mathbb{N}$ is a special clean ring.

While the definitions of von Neumann regularity and $\pi$-regularity extend verbatim from unital rings to the nonunital case, the notions of clean and unit regularity require additional attention in the nonunital situation (since each definition refers to a unit in the given ring). We now show how to naturally extend these latter two notions to rings with local units.

Definition 6 Let $R$ be a ring with local units.

(1) $R$ is called locally unit regular if for each $a \in R$ there is an idempotent $v \in R$ for which $a \in v R v$, and elements $u, u^{\prime} \in v R v$ such that $u u^{\prime}=v=u^{\prime} u$, and $a u a=a$.

(2) $R$ is called locally clean if for each $a \in R$ there is an idempotent $v \in R$ for which $a \in v R v$, and elements $e, u, u^{\prime} \in v R v$ such that $e$ is an idempotent, $u u^{\prime}=v=u^{\prime} u$, and $a=e+u$.

That the two notions given in the previous definition are natural generalizations of the corresponding notions for unital rings is established in the following.

Lemma 3 Let $R$ be a unital ring.

(1) $R$ is locally unit regular if and only if $R$ is unit regular.

(2) $R$ is locally clean if and only if $R$ is clean.

Proof For (1), suppose $R$ is a ring with 1 and is locally unit regular. Let $a \in R$, and let $v, u, u^{\prime}$ as given in the definition. Then $w=u+(1-v)$ and $w^{\prime}=u^{\prime}+(1-v)$ satisfy $w w^{\prime}=1=w^{\prime} w$ and $a=a w a$. Hence $R$ is unit regular. The converse is clear with $v=1$.

Likewise, for (2), suppose $R$ is a ring with 1 and is locally clean. Let $a \in R$, and write $a=u+e$ as given in the definition. Then $a=w+e^{\prime}$, where $e^{\prime}=e+(1-v)$ is an idempotent and $w=u-(1-v)$ is a two-sided unit in $R$ (since with $w^{\prime}=u^{\prime}-(1-v)$, we have $\left.w w^{\prime}=1=w^{\prime} w\right)$. Thus $R$ is clean. As with (1), the converse follows with $v=1$.

Our final result shows that for acyclic graphs $E, L_{K}(E)$ possesses the locally unit regular property, as well as a property involving clean unital subrings. 
Theorem 2 Let $E$ be an arbitrary graph, and let $K$ be any field. Then the following conditions are equivalent:

(1) $E$ is is acyclic.

(2) $L_{K}(E)$ is locally unit regular.

(3) $L_{K}(E)$ is a direct limit of unital strongly clean rings, each of which is special.

Proof $(1) \Leftrightarrow(2)$ Suppose $E$ is acyclic. Then, by Theorem $1, L_{K}(E)$ is a direct union of direct sums of matrix rings each of which, by [9, page 38], is unit regular. It is then clear that $L_{K}(E)$ is locally unit regular, where for each $a \in L_{K}(E)$ we use for $v$ the identity element of the corresponding subring $B(S)$. Conversely, if $L_{K}(E)$ is locally unit regular, then it is, in particular, von Neumann regular. So, by Theorem 1$] E$ is acyclic.

$(2) \Leftrightarrow(3)$ Suppose $L_{K}(E)$ is locally unit regular. Since it is von Neumann regular, Theorem 1 implies that $L_{K}(E)$ is a directed union of direct sums of matrix rings $L_{i}$ each of which, as noted above, is a special clean ring which is, in addition, strongly clean. On the other hand, if $L_{K}(E)$ is a directed union of special clean rings $L_{i}$, then each $L_{i}$ is unit regular by [6, Theorem 1], and so $L_{K}(E)$ is locally unit regular. (Again for each $a \in L_{K}(E)$ we use for $v$ the identity element of the corresponding subring $B(S)$.)

A study of $L_{K}(E)$ for arbitrary graphs $E$ is presented by Goodearl in [8]. Included in [8] is a method to write $E$ as a direct union of countable complete subgraphs. We now show how this approach together with the desingularization process yields an alternate proof of the implication $(3) \Rightarrow(1)$ of Theorem 1 . Our aim in doing so is to contrast this approach with that of using Proposition 1] which helps us to establish not only the von Neumann regularity of $L_{K}(E)$ for an acyclic graph $E$, but uncovers several internal properties of such an $L_{K}(E)$ (e.g., locally matricial and locally unit regular). (Our approach also shows the coincidence of von Neumann regularity with $\pi$-regularity and strong $\pi$-regularity for Leavitt path algebras.) One may also note that the desingularization approach as shown below does not work for $\pi$-regular rings since $\pi$-regularity, unlike von Neumann regularity, is not a Morita invariant (see e.g. 12]). Proposition 1 poses no such restrictions, and provides additional structural insight into these rings.

So suppose $E$ is acyclic. By [8, Proposition 2.7] $L_{K}(E)=\varliminf_{\alpha \in A} L_{K}\left(E_{\alpha}\right)$, with the limit taken over the set $\left\{E_{\alpha} \mid \alpha \in A\right\}$ of countable complete subgraphs of $E$. So in order to show that $L_{K}(E)$ is von Neumann regular, it suffices to show that each $L_{K}\left(E_{\alpha}\right)$ is von Neumann regular, since the direct limit of von Neumann regular rings is von Neumann regular. Since $E$ is acyclic then necessarily so is each $E_{\alpha}$.

Since $E_{\alpha}$ is countable, we may form a desingularization $F_{\alpha}$ of $E_{\alpha}$. (See e.g. 2].) By construction, $F_{\alpha}$ is row-finite. Also, since desingularization preserves Morita equivalence, and von Neumann regularity is preserved by Morita equivalence for rings with local units by [4, Proposition 3.1], it suffices to show that each $L_{K}\left(F_{\alpha}\right)$ is von Neumann regular. Since each $E_{\alpha}$ is acyclic, the desingularization construction shows that each $F_{\alpha}$ is acyclic as well.

But by [5. Lemma 3.2] $F_{\alpha}$ is the direct union of $G_{\beta}$ (the union taken over the set of finite complete subgraphs of $\left.F_{\alpha}\right)$, and $L_{K}\left(F_{\alpha}\right)=\underline{\lim }_{\beta \in B} L_{K}\left(G_{\beta}\right)$. Thus it suffices to show that each $L_{K}\left(G_{\beta}\right)$ is von Neumann regular. Since $G_{\beta}$ is a subgraph of $F_{\alpha}$ we have that $G_{\beta}$ is acyclic. 
So in the end, to establish that $L_{K}(E)$ is von Neumann regular, it suffices to show that for any finite acyclic graph $G$ that $L_{K}(G)$ is von Neumann regular. But by [3, Proposition 3.5] the Leavitt path algebra of a finite acyclic graph is isomorphic to a finite direct sum of finite dimensional matrix rings over the ground field $K$, and such rings are well known to be von Neumann regular (see e.g. [9, Section 1]).

We conclude this article by noting one more consequence of Theorem 1 (we thank the referee for this suggestion). The proof follows directly from the fact that von Neumann regularity is a Morita invariant for rings with local units. We contrast this result with the aforementioned remark that, in general, the $\pi$-regularity property is not a Morita invariant.

Corollary 1 The property of $\pi$-regularity is a Morita invariant for Leavitt path algebras; that is, if $E$ and $F$ are graphs with $L_{K}(E)$ Morita equivalent to $L_{K}(F)$, then $L_{K}(E)$ is $\pi$-regular if and only if $L_{K}(F)$ is $\pi$-regular, and in this case $E$ and $F$ are both acyclic.

\section{acknowledgments}

The authors thank E. Pardo and M. Siles Molina for their valuable discussions during the preparation of this paper. The authors also thank the referee for a very careful reading of, and suggested changes to, the initial version of the manuscript.

\section{References}

1. Abrams, G., Ánh, P.N., Louly, A., Pardo, E.: The classification question for Leavitt path algebras, J. Algebra, to appear. ArXiV: 0706.3874.

2. Abrams, G., Aranda Pino, G.: The Leavitt path algebra of arbitrary graphs, Houston J. Math, to appear.

3. Abrams, G., Aranda Pino, G., Siles Molina, M.: Finite-dimensional Leavitt path algebras, J. Pure Appl. Algebra. 209(3), 753-762 (2007).

4. Ánh, P.N., MÁRKI, L.: Morita equivalence for rings without identity, Tsukuba J. Math. 11(1), 1-16 (1987).

5. Ara, P., Moreno, M.A., Pardo, E.:, Nonstable K-Theory for graph algebras, Algebra Represent. Theory 10(2), 157-178 (2007).

6. Camillo, V., Khurana, D.: A characterization of unit regular rings, Comm. Algebra 29(5), 2293-2295 (2001).

7. Camillo, V., Yu, H.-P.: Stable range one for rings with many idempotents, Trans. A.M.S. 347(8), 3141-3147 (1995).

8. Goodearl, K.: Leavitt path algebras and direct limits, to appear. arXiv:0712.2554 1

9. Goodearl, K.: Von Neumann Regular Rings. Krieger Publ., Malabar, FL (1991). ISBN 0-89464-632-X.

10. Kaplansky, I: Topological representation of algebras. II, Trans. A.M.S. 68(1), 62-75 (1950).

11. Nicholson, W.K, Zhou, Y.: Clean Rings: a survey. In: Advances in Ring Theory: Proceedings of the 4th China-Japan-Korea International Conference, pp. 181-198. World Sci. Publ., Hackensack, N.J. (2005). ISBN: 981-256-425-X.

12. Rowen, L.: Examples of semiperfect rings, Israel J. Math. 65(3), 273-283 (1989).

13. Raeburn, I., Szymański, W.: Cuntz-Krieger algebras of infinite graphs and matrices, Trans. A.M.S. 356(1) , 39-59 (2003). 